\title{
Strain-induced robust magnetic anisotropy and room temperature magnetoelectric coupling effect in epitaxial $\mathrm{SmFeO}_{3}$ film
}

\author{
Jun Zhang ${ }^{1,2,3 \dagger}$, Wuhong Xue ${ }^{1,3 \dagger}$, Tiancong Su ${ }^{1,3}$, Huihui Ji ${ }^{1,3}$, Zhi Yan ${ }^{1,3}$, Guowei Zhou ${ }^{1,3}$, \\ Zhiyong Quan ${ }^{1,3}$ and Xiaohong $\mathrm{Xu}^{1,3^{*}}$
}

\begin{abstract}
Rare-earth orthoferrite $\mathrm{SmFeO}_{3}$ is an outstanding single-phase multiferroic material, holding great potential in novel low-power electronic devices. Nevertheless, simultaneous magnetic and ferroelectric orders as well as magnetoelectric (ME) coupling effect at room temperature (RT) in this system have not been demonstrated yet. In this study, epitaxial $\mathrm{SmFeO}_{3}$ films were successfully prepared onto tensile-strain $\mathrm{Nb}-\mathrm{SrTiO}_{3}$ (Nb-STO) substrates by a pulsed laser deposition (PLD) method. Measurement results show that the films exhibit obvious ferromagnetic and ferroelectric orders at RT. Meanwhile, the magnetic anisotropy gradually changes from out-of-plane (OP) to in-plane (IP) direction with increasing film thickness, which is attributed to the variations of $\mathrm{O} 2 \mathrm{p}$-Fe 3d hybridization intensity and Fe 3dorbit occupancy caused by the strain-relaxed effect. Moreover, electrically driven reversible magnetic switching further proves that the $\mathrm{SmFeO}_{3}$ films exhibit the RT ME coupling effect, suggesting promising applications in new-generation electric-write magnetic-read data storage devices.
\end{abstract}

Keywords: $\mathrm{SmFeO}_{3}$ film, multiferroicity, magnetoelectric coupling, magnetic anisotropy

\section{INTRODUCTION}

Single-phase multiferroics, presenting the coexistence of ferroelectric and magnetic orders simultaneously, have been considered as one class of the most promising materials because of their vast application potential in spintronic devices, information storage, sensors, etc. [1-
6]. However, traditional theory holds that the two orders have incompatible occupation rules and thus lead to extremely rare multiferroic materials in nature [7]. Recently, spin-induced improper ferroelectricity has been widely explored in some oxide materials [8-11]. In such systems, the ferroelectric and magnetic orders bind each other and may exhibit strong magnetoelectric (ME) coupling effect. In order to explain the origin of the magnetically induced ferroelectricity, a large number of theoretical models were proposed one after another, such as reverse Dzyaloshinskii-Moriya (D-M) interaction [12], spin-dependent $p$ - $d$ hybridization and exchange striction mechanism $[13,14]$. So far, numerous single-phase multiferroic materials have been found, such as (Y, Sr, Gd, Dy, Tb, Ho, Lu) $\mathrm{MnO}_{3}$ films [15-18], (Dy, Gd, Tb, Ho, Er, $\mathrm{Lu}) \mathrm{CrO}_{3}$ films [19-23], (Gd, Dy, Yb) $\mathrm{FeO}_{3}$ films [24-26], $\mathrm{EuTiO}_{3}$ films [27], hexaferrite $\mathrm{Ba}_{2-x} \mathrm{Sr}_{x} \mathrm{Mg}_{2} \mathrm{Fe}_{12} \mathrm{O}_{22}$ single crystal [28], and $\mathrm{LaFeO}_{3}$ polycrystal [29]. Despite rich characteristics and fascinating physics, the low working temperature and weak saturation magnetization $\left(M_{\mathrm{s}}\right)$ restrict the practical applications of these materials. In addition, some nanocomposite single-phase systems were also studied. For example, room temperature (RT) multiferroic properties were found in the $\mathrm{PbTiO}_{3}-\mathrm{SmFeO}_{3}$ film and $\mathrm{BiFeO}_{3}-\mathrm{PbTiO}_{3}$ single crystal $[30,31]$, but the $\mathrm{ME}$ coupling effect was rather weak because the magnetic and ferroelectric orders respectively arose from different ions. Therefore, exploring new single-phase multiferroic materials with high temperature, large $M_{\mathrm{s}}$ as well as strong

\footnotetext{
${ }^{1}$ School of Chemistry and Materials Science of Shanxi Normal University \& Key Laboratory of Magnetic Molecules and Magnetic Information Materials of Ministry of Education, Linfen 041004, China

${ }^{2}$ Department of Chemistry \& Chemical Engineering, Lvliang University, Lvliang 033001, China

${ }^{3}$ Research Institute of Materials Science of Shanxi Normal University \& Collaborative Innovation Center for Shanxi Advanced Permanent Magnetic Materials and Techonology, Linfen 041004, China

† These authors contributed equally to this work.

* Corresponding author (email: xuxh@sxnu.edu.cn)
} 
ME coupling effect have become a topical research field.

Parts of rare-earth orthoferrite materials, possess high Neel temperature $\left(T_{\mathrm{N}}\right)$ of around $700 \mathrm{~K}$ and large ME coupling effect at RT, making them the most potential candidates in the microelectronic field. As a typical representative, the hexagon-strained $\mathrm{LuFeO}_{3}$ film with a $\mathrm{PG}_{3} \mathrm{~cm}$ structure has been reported, revealing that the polar structure could persist up to $1050 \mathrm{~K}$, and an antiferromagnetic order was also observed below $440 \mathrm{~K}$, the mechanism might be related to strain-induced asymmetric Lu $5 \mathrm{~d}_{z}{ }^{2}-\mathrm{O} 2 \mathrm{p}_{z}$ hybridization [32]. However, bulk$\mathrm{LuFeO}_{3}$ single crystal with an orthorhombic-stabilized $\mathrm{Pbnm}$ structure lacked such properties. Additionally, orthorhombic $\mathrm{SmFeO}_{3}$ (SFO) was also studied and its bulk single crystal had no ferroelectric order [33], but the film revealed an obvious multiferroic characteristic [34]. Regrettably, the magnetic transition temperature was around $185 \mathrm{~K}$ and below RT, which is the only report on the multiferroic property of the film-style SFO until now to the best of our knowledge. Subsequently, a large number of models have been proposed to explain the ferroelectricity of SFO. Johnson et al. [35] concluded that the exchange striction mechanism was relevant to the manifestation of the improper ferroelectricity. Lee et al. [36] illustrated that the reverse D-M interaction dominated over the exchange striction mechanism in the realization of the improper ferroelectricity. However, recent studies have confirmed that the ferroelectricity should originate from structural distortion [34]. Although many reasonable explanations have been considered, the physical mechanism of ferroelectricity in SFO is still controversial and needs to be further explored.

In this work, we choose SFO material as an object and prepare the films of different thicknesses on the $0.7 \mathrm{wt} \%$ $\mathrm{Nb}-\mathrm{SrTiO}_{3}$ (Nb-STO) substrates which are subjected to an in-plane (IP) tensile strain. The RT ferromagnetic order appears and the magnetic anisotropy gradually transforms from out-of-plane (OP) to IP direction with increasing film thickness. Combining $\mathrm{O} K$-edge $\mathrm{X}$-ray absorption spectra (XAS) with Fe L-edge X-ray linear dichroism (XLD) analyses, we propose that the changes of occupancy state of Fe-3d orbits together with $\mathrm{O} 2 \mathrm{p}-\mathrm{Fe} 3 \mathrm{~d}$ hybridization intensity caused by the strain-relaxed effect, force a tremendous manipulation in the magnetic anisotropy of SFO films. Moreover, the ferroelectricity of the films is confirmed via polarization-electric field (P-E) loops and piezoresponse force microscopy (PFM) phase images. Furthermore, the electrically driven reversible magnetic switching demonstrates an obvious RT ME coupling effect in the SFO films, implying a broad application prospect in the novel generation of magnetoelectricity devices.

\section{EXPERIMENTAL SECTION}

Orthorhombic SFO films with various thicknesses were fabricated onto atomically flat Nb-STO substrates (denoted as Nb-STO/SFO film) by a reflection high-energy electron diffraction-assisted pulsed laser deposition (PLD) system using a $\mathrm{KrF}$ excimer laser $(\lambda=248 \mathrm{~nm})$. During the deposition, the laser energy was stabilized at $350 \mathrm{~mJ}$ and the frequency was repeated at $10 \mathrm{~Hz}$. The dynamic flowing oxygen pressure and substrate temperature were kept at $30 \mathrm{mTorr}(1$ Torr $\approx 133.3 \mathrm{~Pa})$ and $725^{\circ} \mathrm{C}$, respectively. Subsequently, the films were annealed in-situ at the same temperature and an oxygen pressure of 700 Torr for $1.5 \mathrm{~h}$ to further reduce oxygen vacancies and then cooled to $\mathrm{RT}$ at a rate of $15^{\circ} \mathrm{C} / \mathrm{min}$. The crystal orientation and microstructure of the films were determined by X-ray diffraction (XRD) and highangle annular dark-field scanning transmission electron microscopy (HAADF-STEM). The magnetic characterization was performed by using a Quantum Design vibrating sample magnetometer in physical property measurement system (PPMS-VSM). The magnetizationmagnetic field $(M-H)$ loops were obtained with an applied magnetic field of $|H| \leq 15 \mathrm{kOe}$ after the diamagnetic background of the substrates was subtracted. The macroscopic $P$-E loops were measured by Radiant Precision Multiferroic II with a test frequency of $1 \mathrm{kHz}$. All measurements were carried out at RT. Before testing, Au electrodes with a diameter of $300 \mu \mathrm{m}$ were deposited on the films by a magnetron sputtering system using a standard shadow mask, accompanied with optimal sputtering pressure and power of $8 \mathrm{~Pa}$ and $5 \mathrm{~W}$, respectively. The local ferroelectric (magnetic) domain textures were measured using an atomic force microscope (AFM, Bruker, Dimension Icon) equipped with PFM and magnetic force microscopy (MFM) test modules. O K-edge and Fe $L$-edge XAS were performed at beamline 08U1A of Shanghai Synchrotron Radiation Facility (SSRF) in a total electron yield mode. All the $\mathrm{O} K$-edge XAS were collected at an X-ray incident angle perpendicular to the surface of the film. The Fe $L$-edge XAS were measured by selecting two different incident angles of $90^{\circ}\left(E / / a, I_{a b}\right)$ and $30^{\circ}\left(E / / c, I_{c}\right)$ with respect to the film surface, respectively. The XLD corresponds to the difference between IP $(E / / a)$ and OP $(E / / c)$ XAS $\left(I_{a b}-I_{c}\right)$, providing a signal of the preferential orbital occupancy.

\section{RESULTS AND DISCUSSION}

Fig. 1a shows the XRD data of the SFO film deposited on 

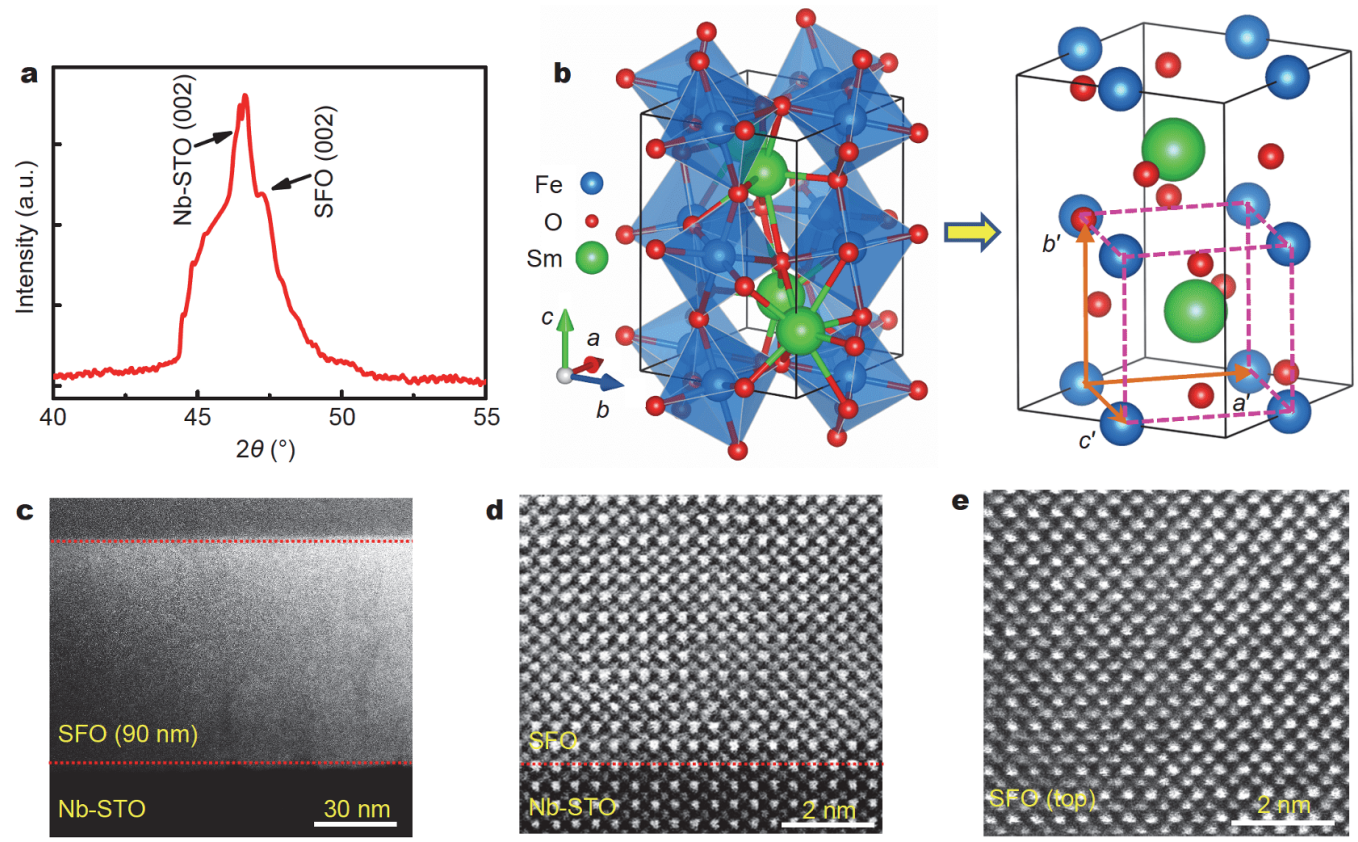

Figure 1 (a) XRD pattern for the SFO film grown on (00l)-oriented Nb-STO single crystal substrate. (b) The orthorhombic crystal structure for bulk SFO unit cell and corresponding perovskite pseudocell. (c) Cross-sectional STEM image of the Nb-STO/SFO $90 \mathrm{~nm}$ film. (d, e) Cross-sectional HAADF-STEM images of the Nb-STO/SFO interface and SFO top surface, respectively.

the (00l) Nb-STO (cubic, $a=3.905 \AA$ ) single crystal substrate. The only perovskite-like pseudocell (002) diffraction peak appears, indicating perfect epitaxial growth along the OP $(00 l)$ orientation. For bulk SFO unit cell, as shown in Fig. 1b, the lattice constants $a=5.400 \AA, b$ $=5.597 \AA$ and $c=7.711 \AA$, correspond to $b^{\prime}=3.856 \AA$, $a^{\prime}=c^{\prime}=3.889 \AA$ of a perovskite pseudocell structure. Thus, it is easy to conclude that the SFO film deposited on the $\mathrm{Nb}$-STO substrate is subjected to the IP tensile strain, which may elongate the IP lattice constant and contract the OP lattice constant of SFO at the interface. As the depositing thickness increases, the restriction of substrate to film gradually weakens due to the strain-relaxed effect, probably causing the lattice constant of SFO to gradually recover the value of the bulk. Fig. 1c presents a lowmagnification cross-sectional STEM image of the asfabricated Nb-STO/SFO film, suggesting that the thickness is about $90 \mathrm{~nm}$. In addition, HAADF-STEM images at the film and substrate interface as well as the top surface in Fig. 1d and e further demonstrate the perfect epitaxial growth of SFO with consistent orientation along the Nb-STO (00l) direction.

As far as we know, most of the single-phase multiferroic materials that have been found at present reveal weak $M_{s}$, which strongly limits their applications. After that, a large number of studies have focused on exploiting new ways of improving the magnetism, such as straininduced displacement of ions or oxygen vacancy ordering $[37,38]$, electrical manipulation of orbital occupancy or spin-orbital coupling $[39,40]$, and element doping-driven change of exchange constant [41], among which the strain effect has proven to be a very powerful approach. Hence, we prepared a series of SFO films with different thicknesses on Nb-STO substrates and measured the IP and OP $M-H$ loops so as to investigate the relationship between magnetism and epitaxial strain, the results are shown in Fig. 2a-c. It is obvious that both $M_{s}$ and magnetic anisotropy of the SFO films display a strong dependence on the film thickness.

For the SFO $30 \mathrm{~nm}$ film, see Fig. 2a, the OP $M-H$ loop exhibits a large $M_{\mathrm{s}}$ value of around $67 \mathrm{emu} \mathrm{cm}^{-3}$. In contrast, the IP direction displays rather weak ferromagnetism, indicating that a robust perpendicular magnetic anisotropy is produced. Previous report also prepared the Nb-STO/SFO $450 \mathrm{~nm}$ film and obtained the OP $M-H$ loop [34]; however, the ferromagnetic transition temperature was only $185 \mathrm{~K}$ (below RT) due to serious structural distortion. As the film thickness reaches up to 60 and $90 \mathrm{~nm}$ (Fig. $2 \mathrm{~b}$ and c), the OP $M_{\mathrm{s}}$ decreases from 44 to $9 \mathrm{emu} \mathrm{cm}{ }^{-3}$ and the IP $M_{\mathrm{s}}$ increases from 16 to $43 \mathrm{emu} \mathrm{cm}^{-3}$, respectively. It is illustrated that a strong magnetic anisotropy rotates from perpendicular to par- 

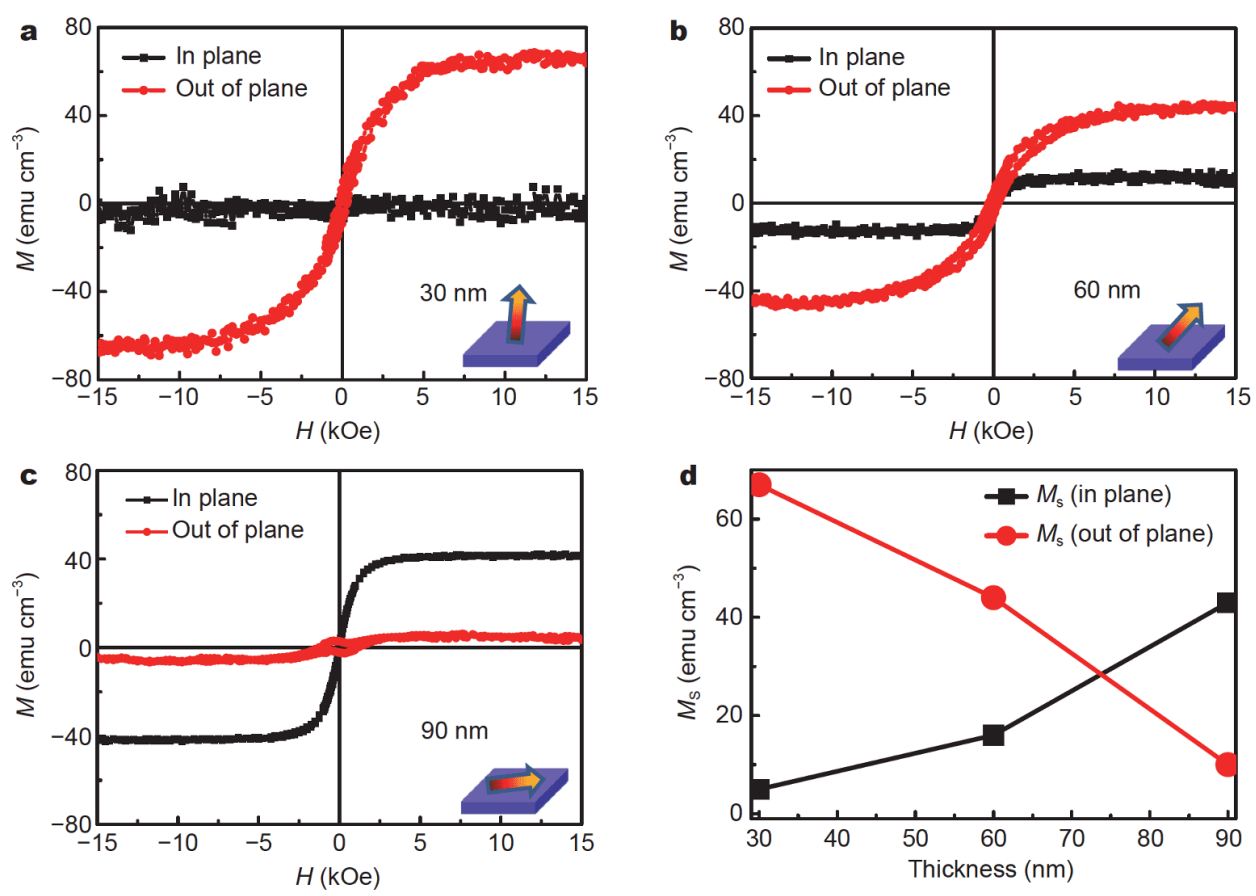

Figure 2 IP (dark line) and OP (red line) $M-H$ loops for the Nb-STO/SFO films with different thicknesses of (a) 30 , (b) 60 and (c) $90 \mathrm{~nm}$. The insets illustrate the direction of magnetic anisotropy. (d) IP and OP $M_{\mathrm{s}}$ values as a function of different thicknesses of 30, $60 \mathrm{and} 90 \mathrm{~nm}$ for the Nb-STO/SFO films.

allel film direction. Fig. $2 \mathrm{~d}$ summarizes the $M_{\mathrm{s}}$ values of all the SFO films, which exhibits a rapid variation along both the IP and OP directions, suggesting that the magnetic moment distributions are indeed sensitive to the film thickness.

To elucidate the influence of thickness on magnetic anisotropy, Fe $L$-edge XLD spectra were measured in all the SFO films at RT. With reference to our previous work [42], we selected the area of $L_{2,3}$ peaks (marked as $A_{\mathrm{XLD}}$ ) to determine the relative orbital occupancy. Negative and positive $A_{\mathrm{XLD}}$ values represent a preferential occupancy of IP $x^{2}-y^{2}$ orbits and OP $3 z^{2}-r^{2}$ orbits, respectively. Fig. $3 a-c$ show Fe XLD spectra with different SFO thicknesses grown on Nb-STO substrates. For the SFO $30 \mathrm{~nm}$ film, the negative $A_{\mathrm{XLD}}$ is remarkable, implying that the IP $x^{2}-y^{2}$ orbit occupancy is stable through a strong tensile strain exerted by the substrate. With the film thickness varying from 30 to 60 and to $90 \mathrm{~nm}$, as shown in Fig. $3 \mathrm{~b}$ and c, negative $A_{\mathrm{XLD}}$ still exists and just the absolute value relatively reduces, which illustrates that preferential occupancy of $x^{2}-y^{2}$ orbits gradually weakens owing to the strain-relaxed effect. Correspondingly, O K-edge XAS were also measured and provided in Fig. 3d. It is obvious that the intensities of $\mathrm{O} 2 \mathrm{p}-\mathrm{Fe} 3 \mathrm{~d}$ hybridization peaks located at the vicinity of $529.6 \mathrm{eV}$ are considerably re- duced as the film thickness increases. Based on the above results, we can conclude that both Fe $x^{2}-y^{2}$ orbital occupation and $\mathrm{O} 2 \mathrm{p}-\mathrm{Fe} 3 \mathrm{~d}$ hybridization intensity are obviously enhanced (weakened) as the film becomes thinner (thicker). Namely, the changes of Fe 3d-orbital occupation together with $\mathrm{O} 2 \mathrm{p}-\mathrm{Fe} 3 \mathrm{~d}$ hybridization may lead to a switch of the magnetic anisotropy from OP to IP direction for the SFO film.

In addition, Fe $L$-edge XAS test was also performed to further confirm the origin of weak ferromagnetism in the SFO film, which shows that the $L_{3}$ peak position of Fe ion located at $708.86 \mathrm{eV}$ (Fig. S1), assigning to an $\mathrm{Fe}^{3+}$ state [43]. This indicates that the SFO films prepared using the PLD method have fewer oxygen vacancies after annealing at a high oxygen pressure of 700 Torr. Therefore, combining with the HAADF-STEM image and XRD pattern, we consider that the weak ferromagnetism of the SFO films is majorly attributed to D-M interaction [36].

Besides the magnetic properties discussed above, macroscopic ferroelectric measurements of $\mathrm{Nb}-\mathrm{STO} / \mathrm{SFO}$ films with different thicknesses were also collected. Fig. S2 displays a simple schematic for measuring the $P-E$ loop and time dependence of the applied voltage-current $(I(V)-t)$ curve. One of the advantages for the Nb-STO/ SFO film is that the Nb-STO substrate can directly serve 

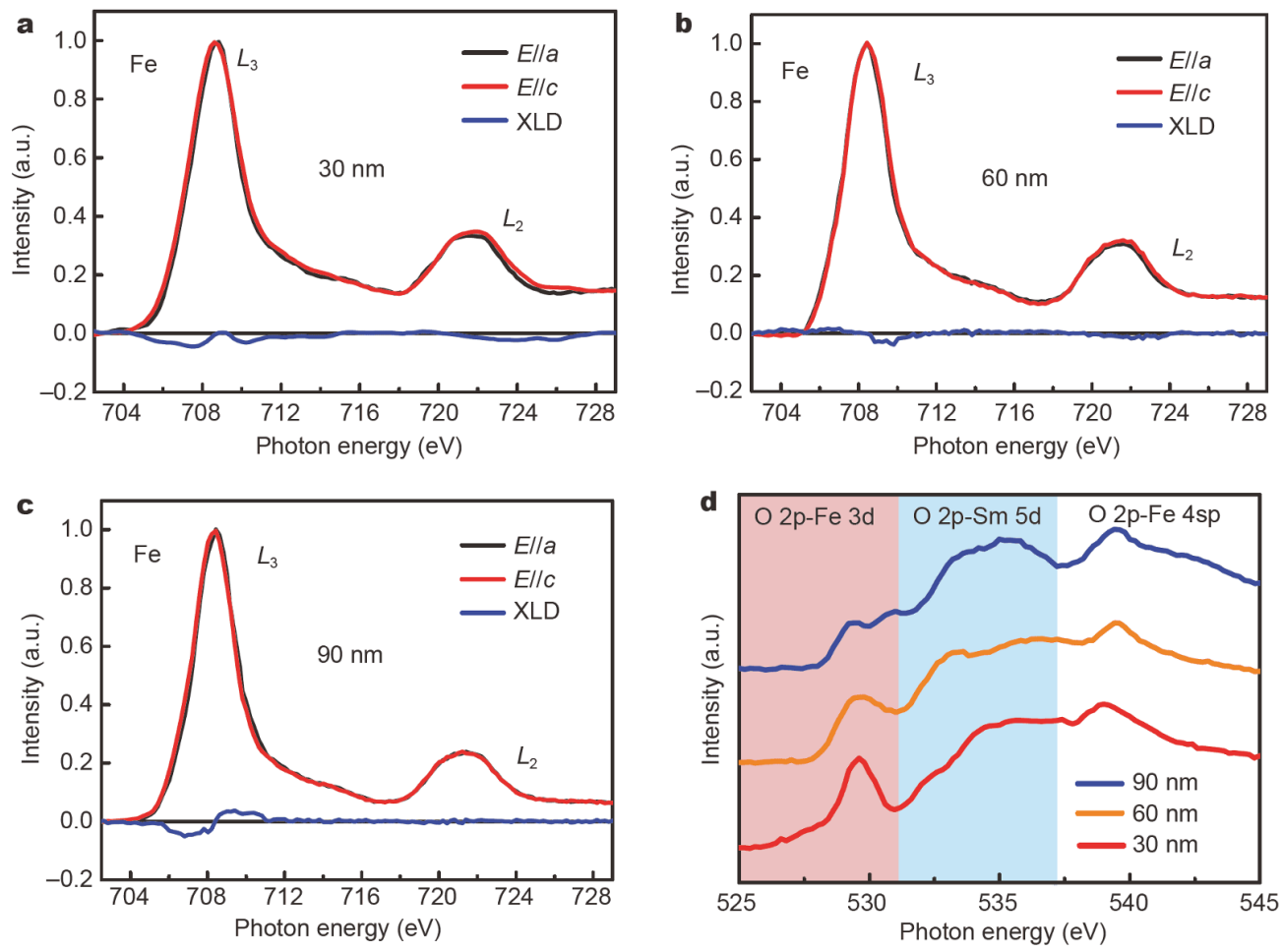

Figure 3 Fe L-edge XLD spectra of Nb-STO/SFO films with different thicknesses of 30 (a), 60 (b) and $90 \mathrm{~nm}$ (c), respectively. (d) O K-edge XAS as a function of the SFO film thickness. The light red, blue and white areas are linked to the O $2 \mathrm{p}$ hybridizing with Fe $3 \mathrm{~d}$, Sm $5 \mathrm{~d}$, and Fe $4 \mathrm{sp}$ state, respectively.

as a bottom electrode, wherein Au electrode with a diameter of $300 \mu \mathrm{m}$ is used as a top electrode. The $P-E$ loops of the SFO $30 \mathrm{~nm}$ film with different maximum test voltages are revealed in Fig. S3a-c, which indicate that all the loops are shown as round whether a small or large voltage is provided. The corresponding triangular-wave $I$ $(V)-t$ curves in Fig. S3d-f imply that an obvious resistance behavior appears instead of ferroelectric behavior because of the huge leakage current. As the film thickness increases to $60 \mathrm{~nm}$ (Fig. S4a-c), all the $P-E$ loops exhibit a cone-shaped form. Although the corresponding $I(V)-t$ curves (Fig. S4d-f) reveal slight hysteresis phenomenon, a large leakage current still exists. When the thickness reaches up to $90 \mathrm{~nm}$ (Fig. 4a), a quasi-rectangular $P$ - $E$ loop with a remanent polarization of $1.1 \mu \mathrm{C} \mathrm{cm}^{-2}$ and a coercive field of $0.2 \mathrm{MV} \mathrm{cm}{ }^{-1}$ can be observed. Meanwhile, the $I(V)-t$ curve (Fig. 4b) exhibits obvious switching and hysteresis characteristics, indicating a clear signal of ferroelectricity. Fig. S5 presents the relationship between the leakage current and applied voltage. The test result shows that the leakage current density remains less than $10^{-5} \mathrm{~A} \mathrm{~cm}^{-2}$ even if it provides a high voltage of $2.5 \mathrm{~V}$. An increasing maximum test voltage such as $3 \mathrm{~V}$ will lead to a large leakage current, which in turn affects the authenticity of the ferroelectric measurement (Fig. S6). To further provide a proof of the local ferroelectricity, OP PFM phase image was characterized by applying a pair of opposite polar voltages. During the measurement, the $4-\mu \mathrm{m}^{2}$ square inside was poled by $+7 \mathrm{~V}$ direct current (DC) voltage after the $16-\mu \mathrm{m}^{2}$ square region outside was poled by $-7 \mathrm{~V}$ DC voltage first, and the result is displayed in Fig. 4c. It is clear that the positive and negative polarized areas show a sharp contrast, suggesting the realization of opposite domain switching. Furthermore, phase-voltage and amplitude-voltage curves were also tested and shown in Fig. 4d, indicating an almost $180^{\circ}$ ferroelectric polarization switching and obvious piezoelectric signal, respectively. Although the explanation has been inconclusive till now, in our case, we suppose the ferroelectricity of SFO film more likely come from the exchange striction mechanism [35].

The RT ME coupling effect is one of the important characteristics for multiferroic materials, as well as the desired goal of our work. To realize the reversible switching of magnetic domains merely by voltage, we applied a series of DC voltages of $0 \mathrm{~V} \rightarrow-1 \mathrm{~V} \rightarrow-2 \mathrm{~V} \rightarrow$ $-3 \mathrm{~V} \rightarrow+0.5 \mathrm{~V}$ on the SFO $90 \mathrm{~nm}$ film via a conductive $\mathrm{Co} / \mathrm{Cr}$-coated tip in AFM. Before the measurement, an 

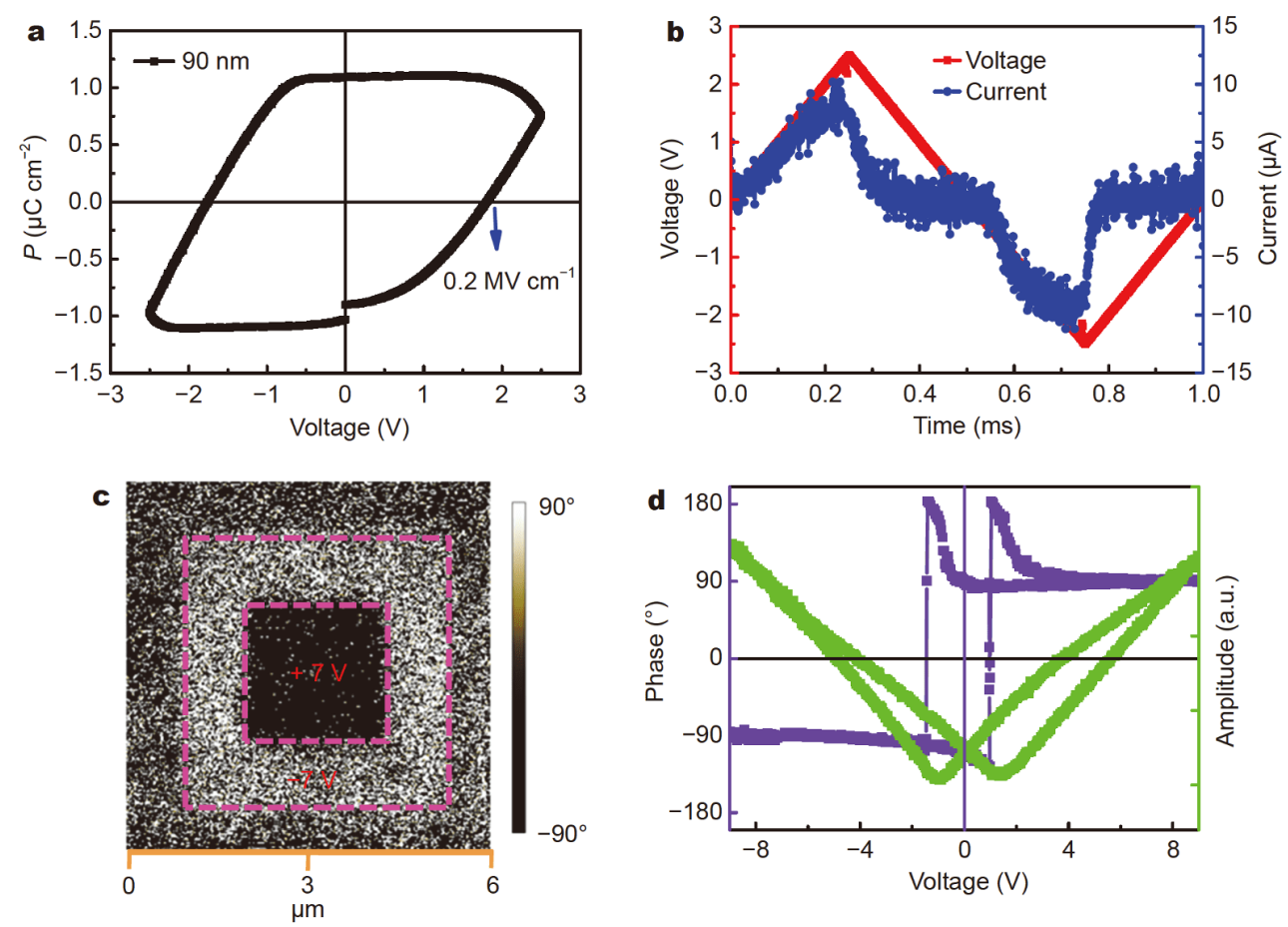

Figure 4 (a) $P$-E loop and (b) $I(V)$ - $t$ curve of the SFO $90 \mathrm{~nm}$ film. (c) The OP PFM phase image of the two boxes after a pair of opposite polar voltages were applied. (d) Phase-voltage and amplitude-voltage curves of the SFO $90 \mathrm{~nm}$ film. The local ferroelectricity of (c) and (d) were measured by using a Pt/Ir-coated conductive tip in the AFM.

oscillation demagnetization treatment was applied to the film. Then, the magnetic domain distributions of the same area were characterized after different voltages were applied. Fig. 5a shows the surface morphology over a $500 \mathrm{~nm} \times 500 \mathrm{~nm}$ scan range and proves a high-quality film with a root-mean-square coarseness of around $0.26 \mathrm{~nm}$. Fig. $5 \mathrm{~b}$ presents the corresponding MFM phase image of the SFO film at a demagnetization state, which shows that the downward-oriented (yellow regions) and upward-oriented (blue regions) mosaic domains are randomly distributed. As the applied voltage varies from 0 to $-2 \mathrm{~V}$, the magnetic domain orientation has some slight variation, but it is not obvious (Fig. $5 \mathrm{c}$ and d). With further increasing voltage to $-3 \mathrm{~V}$, the blue area suddenly increases (Fig. 5e), which means that the majority of downward-oriented magnetic domains reverse to upward

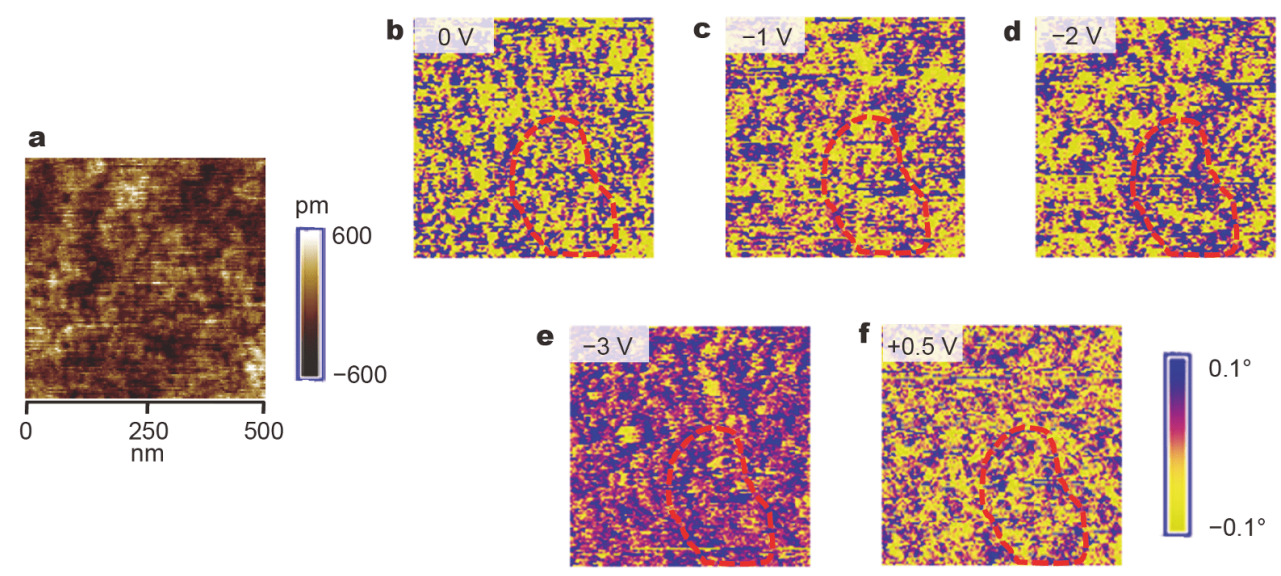

Figure 5 (a) Surface morphology over a $500 \mathrm{~nm} \times 500 \mathrm{~nm}$ scan range in the Nb-STO/SFO $90 \mathrm{~nm}$ film. (b-f) MFM phase images of the SFO film after being subjected to different voltages of $0 \mathrm{~V} \rightarrow-1 \mathrm{~V} \rightarrow-2 \mathrm{~V} \rightarrow-3 \mathrm{~V} \rightarrow+0.5 \mathrm{~V}$. 


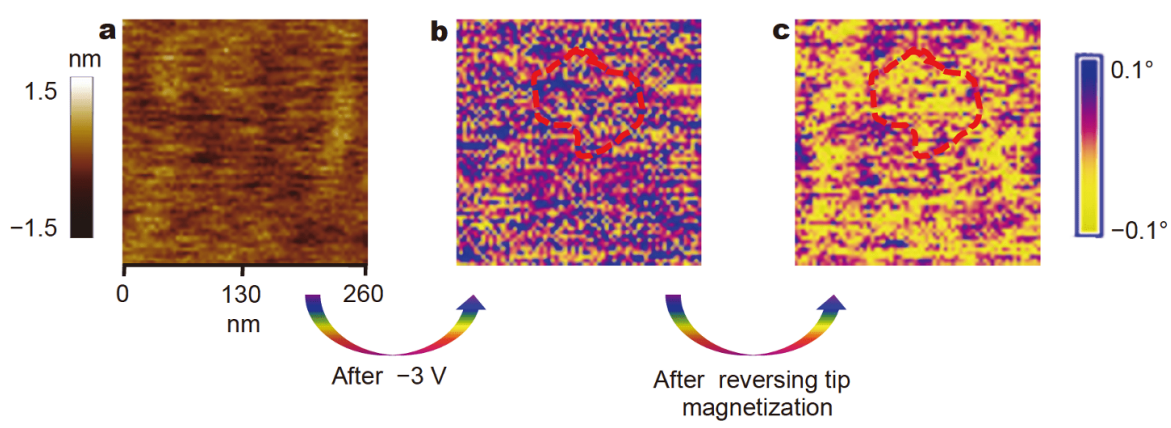

Figure 6 (a) Surface morphology over a $260 \mathrm{~nm} \times 260 \mathrm{~nm}$ scan range in the Nb-STO/SFO $90 \mathrm{~nm}$ film. (b) MFM phase image after applying a $-3 \mathrm{~V}$ voltage. (c) The MFM phase image which was measured by reversing the tip magnetization at $0 \mathrm{~V}$ after the manipulation of (b).

direction at this critical voltage. However, when a small voltage of $+0.5 \mathrm{~V}$ is provided again (Fig. $5 \mathrm{f}$ ), most of the magnetic domains that have been polarized upward reverse to downward direction. It is worth mentioning that the above manipulation shows an asymmetric critical switching voltage, which is probably attributed to the pinning effect of the residual antiferromagnetic-SFO part or different work-function of the used electrodes [44]. Fig. S7 displays the OP PFM phase images of the SFO film with box-in-box patterns written with different voltages and corresponding MFM phase images in the same area. A clear contrast of the ferroelectric and ferromagnetic phases illustrates the ferroelectric domains and the corresponding ferromagnetic domains being electrically switched simultaneously, demonstrating an obvious ME coupling effect.

To verify the authenticity of ME coupling, we also scanned the film by applying the same tip with opposite magnetization. Fig. 6a shows the surface morphology over a $260 \mathrm{~nm} \times 260 \mathrm{~nm}$ range in the $S F O 90 \mathrm{~nm}$ film. When providing a $-3 \mathrm{~V}$ voltage in this area, as revealed in Fig. 6b, the majority of magnetic domains are forced to orientate upward. A pronounced difference in the magnetic domain orientation is observed compared with the same area when measured by reversing the tip magnetization at $0 \mathrm{~V}$ (Fig. 6c). This method can effectively exclude the influence of electrostatic interactions between the film and conductive tip and really prove the electrical control of magnetic phenomenon.

Overall, the present research verifies a controllable magnetic anisotropy by utilizing the epitaxial strain. On the basis of the changes of $\mathrm{O} 2 \mathrm{p}-\mathrm{Fe} 3 \mathrm{~d}$ hybridization intensity and Fe 3d-orbital occupation, we can get a reasonable explanation for the magnetic anisotropy of the SFO film which gradually varies from OP to IP direction with increasing film thickness. A series of ferroelectric tests and electrically controlled magnetic switching give a direct experimental evidence for the existence of multiferroicity as well as ME coupling effect at RT.

\section{CONCLUSIONS}

Fully epitaxial Nb-STO/SFO films were successfully prepared by using the PLD method. Measurement results illustrate that the SFO films exhibit obvious ferromagnetic and ferroelectric orders at RT, and the magnetic anisotropy gradually changes from OP to IP direction with increasing film thickness. We propose that the variation of magnetic anisotropy direction is attributed to the changes of Fe 3d-orbital occupation and $\mathrm{O} 2 \mathrm{p}$ $\mathrm{Fe} 3 \mathrm{~d}$ hybridization intensity due to the strain-relaxed effect. Moreover, macroscopic P-E loop, local PFM phase image as well as phase (amplitude)-voltage curves simultaneously show that the films are ferroelectric. Furthermore, electrically driven reversible magnetic switching further confirms that the films exhibit RT ME coupling effect, which is promising for applications in ferroelectric random access memory (FeRAM) and other electronic devices.

Received 20 March 2020; accepted 12 May 2020;

published online 14 August 2020

1 Kimura T, Goto T, Shintani H, et al. Magnetic control of ferroelectric polarization. Nature, 2003, 426: 55-58

2 Yoong HY, Wang $\mathrm{H}$, Xiao J, et al. Tunneling electroresistance effect in ultrathin $\mathrm{BiFeO}_{3}$-based ferroelectric tunneling junctions. Appl Phys Lett, 2016, 109: 242901

3 Bibes M, Barthélémy A. Towards a magnetoelectric memory. Nat Mater, 2008, 7: 425-426

4 Ma J, Hu J, Li Z, et al. Recent progress in multiferroic magnetoelectric composites: From bulk to thin films. Adv Mater, 2011, 23: 1062-1087

5 Zhang Y, Xue F, Chen Z, et al. Presence of a purely tetragonal phase in ultrathin $\mathrm{BiFeO}_{3}$ films: Thermodynamics and phase-field simulations. Acta Mater, 2020, 183: 110-117

6 Guo R, You L, Zhou Y, et al. Non-volatile memory based on the ferroelectric photovoltaic effect. Nat Commun, 2013, 4: 1990 
7 Hill NA. Why are there so few magnetic ferroelectrics? J Phys Chem B, 2000, 104: 6694-6709

8 Kocsis V, Nakajima T, Matsuda M, et al. Magnetization-polarization cross-control near room temperature in hexaferrite single crystals. Nat Commun, 2019, 10: 1247

9 Zhai K, Shang DS, Chai YS, et al. Room-temperature nonvolatile memory based on a single-phase multiferroic hexaferrite. Adv Funct Mater, 2018, 28: 1705771

10 Komabuchi M, Urushihara D, Kimata $\mathrm{Y}$, et al. Multiaxial magnetocrystalline anisotropy in the X-type hexaferrite $\mathrm{Sr}_{2} \mathrm{Co}_{2} \mathrm{Fe}_{28} \mathrm{O}_{46}$ at low temperature. Phys Rev B, 2019, 100: 094406

11 Chmiel FP, Prabhakaran D, Steadman P, et al. Magnetoelectric domains and their switching mechanism in a Y-type hexaferrite. Phys Rev B, 2019, 100: 104411

12 Sergienko IA, Dagotto E. Role of the Dzyaloshinskii-Moriya interaction in multiferroic perovskites. Phys Rev B, 2006, 73: 0944343

13 Choi YJ, Yi HT, Lee S, et al. Ferroelectricity in an Ising chain magnet. Phys Rev Lett, 2008, 100: 047601

14 Tokura Y, Seki S, Nagaosa N. Multiferroics of spin origin. Rep Prog Phys, 2014, 77: 076501

15 Lueken $\mathrm{H}$. A magnetoelectric effect in $\mathrm{YMnO}_{3}$ and $\mathrm{HoMnO}_{3}$. Angew Chem Int Ed, 2008, 47: 8562-8564

16 Guo JW, Wang PS, Yuan Y, et al. Strain-induced ferroelectricity and spin-lattice coupling in $\mathrm{SrMnO}_{3}$ thin films. Phys Rev B, 2018, 97: 235135

17 Mukherjee S, Shimamoto K, Windsor YW, et al. Multiferroic phase diagram of E-type $\mathrm{RMnO}_{3}$ films studied by neutron and X-ray diffraction. Phys Rev B, 2018, 98: 174416

18 Fedorova NS, Windsor YW, Findler C, et al. Relationship between crystal structure and multiferroic orders in orthorhombic perovskite manganites. Phys Rev Mater, 2018, 2: 104414

19 McDannald A, Vijayan S, Shi J, et al. Magnetic and tunable dielectric properties of $\mathrm{DyCrO}_{3}$ thin films. J Mater Sci, 2019, 54: 8984-8994

20 Ghosh A, Pal A, Dey K, et al. Atypical multiferroicity of $\mathrm{HoCrO}_{3}$ in bulk and film geometry. J Mater Chem C, 2015, 3: 4162-4167

21 Meher KRSP, Martin C, Caignaert V, et al. Multiferroics and magnetoelectrics: A comparison between some chromites and cobaltites. Chem Mater, 2014, 26: 830-836

22 Albargi H, Alqahtani M, Blythe HJ, et al. Magnetic and magnetooptical properties of films of multiferroic $\mathrm{GdMnO}_{3}$ grown on LSAT $\left[\left(\mathrm{LaAlO}_{3}\right)_{0.3}\left(\mathrm{Sr}_{2} \mathrm{AlTaO}_{6}\right)_{0.7}\right]$ (100) and (111). Thin Solid Films, 2018, 645: 326-333

23 Glavic A, Becher C, Voigt J, et al. Stability of spin-driven ferroelectricity in the thin-film limit: Coupling of magnetic and electric order in multiferroic $\mathrm{TbMnO}_{3}$ films. Phys Rev B, 2013, 88: 054401

24 Tokunaga Y, Furukawa N, Sakai H, et al. Composite domain walls in a multiferroic perovskite ferrite. Nat Mater, 2009, 8: 558-562

25 Tokunaga $\mathrm{Y}$, Iguchi $\mathrm{S}$, Arima $\mathrm{T}$, et al. Magnetic-field-induced ferroelectric state in $\mathrm{DyFeO}_{3}$. Phys Rev Lett, 2008, 101: 097205

26 Jeong YK, Lee JH, Ahn SJ, et al. Structurally tailored hexagonal ferroelectricity and multiferroism in epitaxial $\mathrm{YbFeO}_{3}$ thin-film heterostructures. J Am Chem Soc, 2012, 134: 1450-1453

27 Lee JH, Fang L, Vlahos E, et al. A strong ferroelectric ferromagnet created by means of spin-lattice coupling. Nature, 2010, 466: 954958

28 Zhai K, Wu Y, Shen S, et al. Giant magnetoelectric effects achieved by tuning spin cone symmetry in Y-type hexaferrites. Nat Commun, 2017, 8: 519

29 Acharya S, Mondal J, Ghosh S, et al. Multiferroic behavior of lanthanum orthoferrite $\left(\mathrm{LaFeO}_{3}\right)$. Mater Lett, 2010, 64: 415-418

30 Wang $\mathrm{Y}$, Zhao $\mathrm{H}$, Zhang L, et al. Well-saturated ferroelectric polarization in $\mathrm{PbTiO}_{3}-\mathrm{SmFeO}_{3}$ thin films. Inorg Chem Front, 2016, 3: $1473-1479$

31 Zhuang J, Bokov AA, Zhang N, et al. Single crystal growth and hierarchical ferroelectric domain structure of $(1-x) \mathrm{BiFeO}_{3}-$ $x \mathrm{PbTiO}_{3}$ solid solutions. Cryst Growth Des, 2018, 18: 4503-4510

32 Wang $\mathrm{W}$, Zhao J, Wang W, et al. Room-temperature multiferroic hexagonal $\mathrm{LuFeO}_{3}$ films. Phys Rev Lett, 2013, 110: 237601

33 Kuo CY, Drees Y, Fernández-Díaz $\mathrm{M}$, et al. $k=0$ magnetic structure and absence of ferroelectricity in $\mathrm{SmFeO}_{3}$. Phys Rev Lett, 2014, 113: 217203

34 Cheng $\mathrm{Z}$, Hong $\mathrm{F}$, Wang $\mathrm{Y}$, et al. Interface strain-induced multiferroicity in a $\mathrm{SmFeO}_{3}$ film. ACS Appl Mater Interfaces, 2014, 6: 7356-7362

35 Johnson RD, Terada N, Radaelli PG. Comment on "spin-cantinginduced improper ferroelectricity and spontaneous magnetization reversal in $\mathrm{SmFeO}_{3}$ ". Phys Rev Lett, 2012, 108: 219701

36 Lee JH, Jeong YK, Park JH, et al. Spin-canting-induced improper ferroelectricity and spontaneous magnetization reversal in $\mathrm{SmFeO}_{3}$. Phys Rev Lett, 2011, 107: 117201

37 Zhang J, Chen X, Zhang Q, et al. Magnetic anisotropy controlled by distinct interfacial lattice distortions at the $\mathrm{La}_{1-x} \mathrm{Sr}_{x} \mathrm{CoO}_{3} /$ $\mathrm{La}_{2 / 3} \mathrm{Sr}_{1 / 3} \mathrm{MnO}_{3}$ interfaces. ACS Appl Mater Interfaces, 2018, 10: 40951-40957

38 Mehta VV, Biskup N, Jenkins C, et al. Long-range ferromagnetic order in $\mathrm{LaCoO}_{3-\delta}$ epitaxial films due to the interplay of epitaxial strain and oxygen vacancy ordering. Phys Rev B, 2015, 91: 144418

39 Cui B, Song C, Gehring GA, et al. Electrical manipulation of orbital occupancy and magnetic anisotropy in manganites. Adv Funct Mater, 2015, 25: 864-870

40 Zhao S, Wang L, Zhou Z, et al. Ionic liquid gating control of spin reorientation transition and switching of perpendicular magnetic anisotropy. Adv Mater, 2018, 30: 1801639

$41 \mathrm{Hu}$ S, Cazorla C, Xiang F, et al. Strain control of giant magnetic anisotropy in metallic perovskite $\mathrm{SrCoO}_{3-\delta}$ thin films. ACS Appl Mater Interfaces, 2018, 10: 22348-22355

42 Zhou G, Yan Z, Bai Y, et al. Exchange bias effect and orbital reconstruction in (001)-oriented $\mathrm{LaMnO}_{3} / \mathrm{LaNiO}_{3}$ superlattices. ACS Appl Mater Interfaces, 2017, 9: 39855-39862

43 Agui A, Mizumaki M, Kuroda T, et al. Temperature and polarization dependence of $\mathrm{Fe} L_{3}$-edge X-ray absorption spectra of $\mathrm{LuFe}_{2} \mathrm{O}_{4}$. J Electron Spectr Related Phenomena, 2014, 197: 13-16

44 Gich M, Fina I, Morelli A, et al. Multiferroic iron oxide thin films at room temperature. Adv Mater, 2014, 26: 4645-4652

Acknowledgements This work was supported by the National Natural Science Foundation of China (51871137, 51901118, 51571136 and 61904099). The authors acknowledge Shanghai Synchrotron Radiation Facility at the Beamline BL08U1A and the National Synchrotron Radiation Laboratory at the Beamline BL12-a for the XAS measurements.

Author contributions Zhang J and Xue $\mathrm{W}$ designed the experiments. Zhang J wrote the manuscript. Su T and Ji H performed the experiments. Yan Z, Zhou G and Quan Z helped analyze the results. Xu X supervised the work and revised the manuscript. All authors contributed to the general discussion.

Conflict of interest The authors declare that they have no conflict of interest. 
Supplementary information online version of the paper.

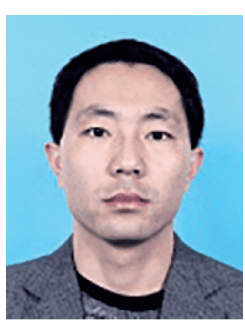

Jun Zhang received his MS degree from Shanxi Normal University in 2011. He is now a PhD candidate under the supervision of Prof. Xiaohong $\mathrm{Xu}$ at the Research Institute of Materials Science of Shanxi Normal University. His current research interest focuses on the ferroelectric and multiferroic behaviors in perovskite complex oxide films and heterostructures.

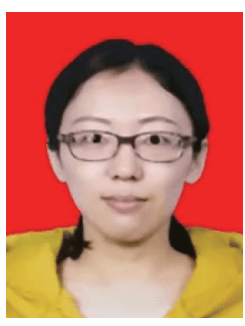

Wuhong Xue obtained her $\mathrm{PhD}$ degree from Shanxi Normal University in 2018. She is currently a lecturer in Prof. Xu's group at Shanxi Normal University. Her current research interest focuses on the preparation of two-dimensional materials and investigations on their magnetoelectric properties.

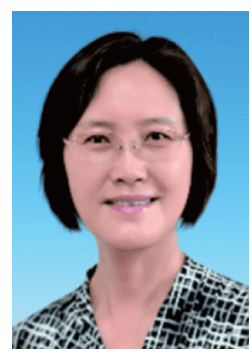

Xiaohong $\mathbf{X u}$ received her $\mathrm{PhD}$ degree in material science and engineering from Xi'an Jiaotong University, China, in 2001. From 2001 to 2006, she was in Huazhong University of Science and Technology, China, the University of Sheffield, UK, and Tohoku University, Japan, as a postdoc or research fellow. Her research interest includes oxide semiconductor spintronics, magnetic recording media and interface physics of heterostructures. She is a Distinguished Young Scholar awarded by the National Natural Science Foundation of China.
应力诱导外延 $\mathrm{SmFeO}_{3}$ 薄膜强烈的磁各向异性以 及室温磁电耦合效应

张军 $^{1,2,3 \dagger}$, 薛武红 ${ }^{1,3 \dagger}$, 苏天聪, 姬慧慧 ${ }^{1,3}$, 严志 ${ }^{1,3}$, 周国伟 ${ }^{1,3}$,
全志勇, $^{1,3}$, 许小红

摘要 稀土正铁氧体 $\mathrm{SmFeO}_{3}$ 由于在低能耗自旋电子学器件领域的 潜在应用, 被认为是一种杰出的单相多铁材料. 然而, 至今尚未证明 该体系在室温下兼具磁性、铁电性以及磁电耦合效应. 本文使用 脉冲激光沉积法在可以提供拉应力的 $\mathrm{Nb}-\mathrm{SrTiO}_{3}$ 基片上成功外延 了倾斜反铁磁 $\mathrm{SmFeO}_{3}$ 单层薄膜. 测量结果显示薄膜具有明显的室 温铁磁和铁电序. 同时, 随着厚度的增加, 薄膜的磁各向异性逐渐 从面外转移到面内方向, 这是由应力释放效应导致的 $\mathrm{O} 2 \mathrm{p}-\mathrm{Fe} 3 \mathrm{~d}$ 杂 化强度和Fe-3d轨道占据变化同时引起的. 另外, 电压驱动可逆磁畴 反转进一步证明 $\mathrm{SmFeO}_{3}$ 薄膜具有明显的室温磁电耦合效应. 本工 作对设计新一代电写磁读数据存储器件具有一定的推动作用. 